\title{
ERRATUM
}

\section{Rotating planar gravity currents at moderate Rossby numbers: fully resolved simulations and shallow-water modelling - ERRATUM}

\author{
Jorge S. Salinas, Thomas Bonometti, Marius Ungarish \\ and Mariano I. Cantero
}

doi:10.1017/jfm.2019.152, Published by Cambridge University Press, 20 March 2019

First, we wish to clarify that in equations (3.14)-(3.15) of Salinas et al. (2019) all variables are dimensional except for the angular velocity $\omega$ and the coefficient $k$. After (3.15), we switch to dimensionless variables for the streamwise direction $x$, the front location $x_{N}$, the Ekman layer $\delta_{E}$, the local height $h$ (scaled by the initial height $h_{0}$ ) and time $t$ (scaled by $1 / \Omega$, with $\Omega$ the angular velocity of the rotating system).

Therefore, equations (3.18)-(3.19) must be corrected to

$$
\begin{gathered}
\mathcal{C}^{2} \frac{\mathrm{d}}{\mathrm{d} t}\left[(2+\omega) \omega x_{N}^{4}\right]=2 k E^{1 / 2} \omega x_{N}^{2}, \\
\frac{\mathrm{d} \hat{\omega}}{\mathrm{d} t}=-\left[2 k\left(\frac{3}{2}\right)^{1 / 3} x_{0}^{-2 / 3} \mathcal{C}^{-2 / 3} E^{1 / 2}\right] \frac{\hat{\omega}^{5 / 3}}{1+\hat{\omega}}=-K_{s u} \frac{\hat{\omega}^{5 / 3}}{1+\hat{\omega}},
\end{gathered}
$$

where $\hat{\omega}=-\omega, x_{0}$ is the initial location of the front in the streamwise direction and $K_{s u}$ is the spin-up constant of the model without mixing (see expression in brackets in (2)). Recall that $\mathcal{C}$ and $E$ are the Coriolis and Ekman numbers, respectively.

Second, note the change of $K_{s u}$. The corrected value of $K_{s u}$ introduces corrections in the last column of table 2 (see below). Also, the corrected value of $K_{s u}$ motivates the replacement of figure 14 of the original paper with figure 1 below. As for $K_{s u}^{m}-$ the spin-up constant of the model with turbulent mixing - it should be stressed that even if its expression remains unchanged (equation (3.21)), the value of $K_{s u}^{m}$ is modified due to the change of $K_{s u}$. The corrected value of $K_{s u}$ and $K_{s u}^{m}$ introduces corrections in the last column of table 1 .

Third, point (3) in $\S 5$ of the original paper should also be changed. Overall, the corrected (3.19) yields fair agreement between the spin-up SW no-mixing predictions and the DNS results, in particular for large Sc. Conversely, the corrected mixing model (at least in its present form) overestimates the mean drift velocity of the slow expanding front for the range of parameters of this investigation.

The good performance of the spin-up model without mixing in the present flow merits attention, but a conclusive understanding of this effect is beyond the scope of this erratum. A plausible explanation is that the local Richardson number $R i$ at the interface is not small during the spin-up, and hence the mixing and momentum 

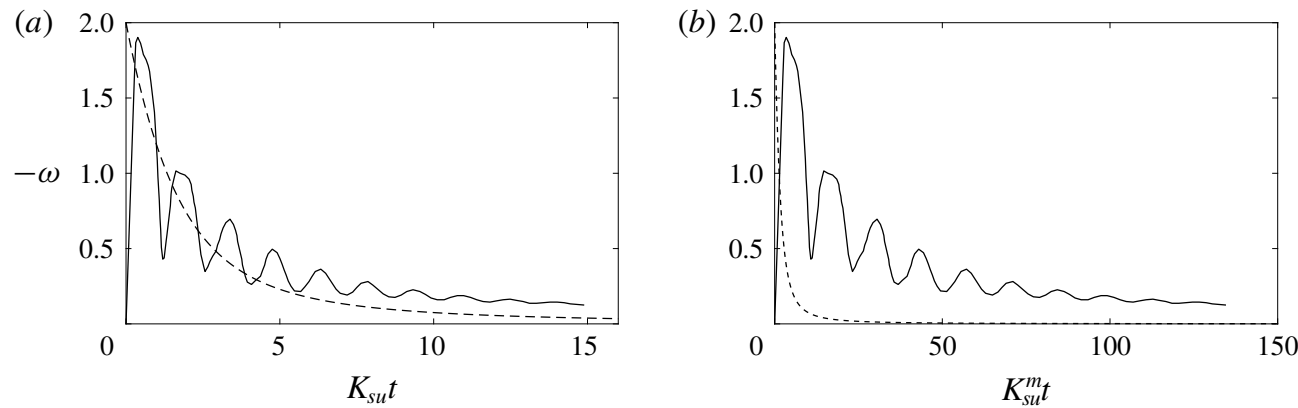

FIGURE 1. Spin-up $-\omega=-\tilde{v} /(\mathcal{C} \tilde{x})$ as a function of (a) $K_{s u} t$ and $(b) K_{s u}^{m} t$. Parameter $t$ is scaled with $1 / \Omega$ (here $t=\mathcal{C} \tilde{t}$ ): SW theoretical model (dashed line) (a) without mixing and corrected $K_{s u}$ and $(b)$ with mixing and corrected $K_{s u}^{m}$; DNS, maximum angular velocity for case S1-C15-N (solid line).

$\begin{array}{lcclccc} & \text { Approach } & \text { Reference } & \mathcal{C} & S c & B C & \mathrm{~d} \bar{x}_{F} / \mathrm{d} \tilde{t} \\ \text { Increasing } \mathcal{C} & \text { DNS } & \text { S1-C10-F } & 0.1 & 1 & F S & 1.6 \times 10^{-3} \\ & \text { DNS } & \text { S1-C15-F } & 0.15 & 1 & F S & 1.0 \times 10^{-3} \\ & \text { DNS } & \text { S1-C25-F } & 0.25 & 1 & F S & 7.0 \times 10^{-4} \\ & \text { SW } & \text { (no mixing) } & 0.1 & & F S & 2.4 \times 10^{-2} \\ & \text { SW } & \text { (no mixing) } & 0.15 & & F S & 1.7 \times 10^{-2} \\ & \text { SW } & \text { (no mixing) } & 0.25 & & F S & 1.1 \times 10^{-2} \\ & \text { SW } & \text { (mixing) } & 0.1 & & F S & 9.1 \times 10^{-2} \\ & \text { SW } & \text { (mixing) } & 0.15 & & F S & 6.2 \times 10^{-2} \\ & \text { SW } & \text { (mixing) } & 0.25 & & F S & 3.9 \times 10^{-2} \\ \text { Increasing Sc } & \text { DNS } & \text { S1-C15-F } & 0.15 & 1 & F S & 1.0 \times 10^{-3} \\ \text { (without wall friction) } & \text { DNS } & \text { S5-C15-F } & 0.15 & 5 & F S & 3.0 \times 10^{-3} \\ & \text { DNS } & \text { SI-C15-F } & 0.15 & \infty & F S & 9.3 \times 10^{-3} \\ & \text { SW } & \text { (no mixing) } & 0.15 & & F S & 1.7 \times 10^{-2} \\ & \text { SW } & \text { (mixing) } & 0.15 & & F S & 6.2 \times 10^{-2} \\ & \text { DNS } & \text { S1-C15-N } & 0.15 & 1 & N S & 7.6 \times 10^{-3} \\ \text { Increasing Sc } & \text { DNS } & \text { S5-C15-N } & 0.15 & 5 & N S & 1.2 \times 10^{-2} \\ \text { (with wall friction) } & \text { DNS } & \text { SI-C15-N } & 0.15 & \infty & N S & 1.7 \times 10^{-2} \\ & \text { SW } & \text { (no mixing) } & 0.15 & & N S & 3.5 \times 10^{-2} \\ & \text { SW } & \text { (mixing) } & 0.15 & & N S & 9.9 \times 10^{-2}\end{array}$

TABLE 1. Mean 'drift' velocity $\mathrm{d} \bar{x}_{F} / \mathrm{d} \tilde{t}$ of the slow expanding front (computed for $\tilde{t} \geqslant 100$ ) corresponding to the slope of the dashed lines in figures 2 and 9. DNS refers to the fully resolved simulations (see table 1 of the original paper) and SW refers to the corrected shallow-water theoretical model without mixing (2) and with mixing (3.21). FS and NS refer to free slip and no slip for DNS, and to $k=1 / 2$ and $3 / 2$ (see $\S 3.3$ for definition) for SW, respectively. Note that the DNS value (run SI-C15-N) corrects a misprint in the original paper. 
Rotating gravity currents at moderate Rossby number - ERRATUM 891 E1-3

transfer (drag) are small, making the classical Ekman layer a good approximation. We estimate $R i$ as follows:

$$
R i=\frac{\frac{g}{\rho_{0}}\left|\frac{\partial \rho}{\partial z}\right|}{\left(\frac{\partial u}{\partial z}\right)^{2}} \approx \frac{g^{\prime} / \delta_{\rho}}{\left(V / \delta_{V}\right)^{2}},
$$

where $\rho$ and $u$ are the local density and velocity, respectively, $z$ is the vertical coordinate, $\rho_{0}$ is the density of the ambient fluid, $g^{\prime}$ is the reduced gravity, $\delta_{\rho}\left(\delta_{V}\right)$ is the characteristic thickness of the density (momentum) transition layer at the interface between the current and the ambient and $V$ is the characteristic speed difference across $\delta_{V}$. We approximate $\delta_{\rho}, \delta_{V}$ and $V$ as

$$
\delta_{\rho} \approx \frac{h_{0}}{\sqrt{\operatorname{ReSc}}}, \quad \delta_{V} \approx h_{0} \sqrt{E}, \quad V \approx \Omega h_{0} x_{0} \hat{\omega} x_{N},
$$

where $R e$ is the Reynolds number, $S c$ is the Schmidt number and $x_{0}, \hat{\omega}$ and $x_{N}$ are dimensionless. Note that the scaling law for $\delta_{\rho}$ was confirmed in the case of non-rotating gravity currents, in the same range of $R e$ and $S c$ as here, by the DNS of Bonometti \& Balachandar (2008); see, for example, their figure 17. The local Richardson number thus reads (dimensional variables)

$$
R i \approx E \sqrt{\operatorname{ReSc}} \frac{g^{\prime} h_{0}}{\left(\hat{\omega} x_{N}\right)^{2}} \frac{1}{\left(\Omega h_{0} x_{0}\right)^{2}},
$$

which can be written using the definition of $\mathcal{C}$ as

$$
R i \approx E \sqrt{\operatorname{ReSc}} \frac{\mathcal{C}^{-2}}{\left(\hat{\omega} x_{N}\right)^{2}} \frac{1}{x_{0}^{2}},
$$

where all variables are now dimensionless. Finally, we use (3.13) and (3.17) to eliminate $E$ and $x_{N}$, respectively, and obtain our estimate of $R i$ as

$$
R i \approx\left(\frac{3}{2} x_{0}^{4}\right)^{-2 / 3} R e^{-1 / 2} S c^{1 / 2} \mathcal{C}^{-5 / 3} \hat{\omega}^{-4 / 3}
$$

During spin-up the estimated $R i$ increases because $\hat{\omega}$ decreases, and for the values of $R e, S c$ and $\mathcal{C}$ of our DNS the typical $R i$ is larger than 0.25 . We also note that a sharp density transition layer enhances stability, and indeed the spin-up model without mixing shows better agreement for DNS with large $S c$.

\section{REFERENCES}

Bonometti, T. \& BalachandaR, S. 2008 Effect of Schmidt number on the structure and propagation of density currents. Theor. Comput. Fluid Dyn. 22 (5), 341-361.

Salinas, J. S., Bonometti, T., Ungarish, M. \& CAntero, M. I. 2019 Rotating planar gravity currents at moderate Rossby numbers: fully resolved simulations and shallow-water modelling. J. Fluid Mech. 867, 114-145. 